Nuclear Physics A410 (1983) 103-124

(C) North-Holland Publishing Company

\title{
SHELL-MODEL COULOMB ENERGIES
}

\author{
E. COMAY and J. JÄNECKE \\ Department of Physics, The University of Michigan, Ann Arbor, Michigan 48109, USA
}

Received 6 April 1983

(Revised 6 June 1983)

\begin{abstract}
Anaiytical expressions have been derived for the total Coulomb energies, for isoscalar, isovector and isotensor Coulomb energies, and for Coulomb energy differences of nuclei with protons and neutrons in equivalent and in different major shell regions. The approach is related to earlier theoretical treatments of Carlson and Talmi and of Hecht. A simple $A$-dependence is assumed to account for the increase in radius. Introducing average matrix elements for the Coulomb interactions between valence-valence, valence-core, and core-core protons in each of the six major shell regions as adjustable parameters, a global fit with 21 parameters to 288 experimental Coulomb displacement energies with $4<A<240$ gives good agreement with a standard deviation of $\sigma=45 \mathrm{keV}$. The interaction energies display discontinuities particularly at major proton shell crossings, and they generally decrease within shells less rapidly than $A^{-1 / 3}$. The average decrease, however. follows approximately an $A^{-1 / 3}$ dependence which explains the success of Coulomb energy equations based on liquid-drop models.
\end{abstract}

\section{Introduction}

The investigation of the electrostatic interaction between the protons inside a nucleus has long been used to obtain nuclear-structure information ${ }^{1-3}$ ). The discovery of isobaric analog states in medium-heavy and heavy nuclei ${ }^{4,5}$ ) has led to a significantly improved understanding.

The discussion of the electrostatic interaction and related effects has three main aspects: (i) From a liquid-drop-model point of view, the Coulomb energy depends on the size and shape of a nucleus ${ }^{2,3}$ ). Studies of isotope shift and deformation effects are still carried out today ${ }^{6,7}$ ). (ii) From a shell-model point of view, the Coulomb energy of a nucleus results from the contributions of interacting proton pairs ${ }^{8-10}$ ). Pairing effects, the dependence on shell-model orbits and their size factors are important considerations. (iii) The significance of exchange effects ${ }^{2,3}$ ) was recognized early. Numerous other small but important effects such as the charge dependence of the strong force, the electromagnetic spin-orbit interaction, isospin mixing, the Thomas-Ehrman shift, etc. contribute to the Coulomb displacement energies and have become the subject of detailed studies ${ }^{11,12}$ ). Open questions still remain.

The present work concentrates on shell-model considerations. It follows closely the theoretical treatments of Carlson and Talmi ${ }^{8}$ ) and of Hecht ${ }^{9,10}$ ) but includes also regions of nuclei where protons and neutrons occupy shell-model orbits from 
different major shells. It was hoped that a global numerical analysis of all known Coulomb displacement energies based on the equations of this work would yield information about Coulomb interaction energies. Also, a few-parameter shellmodel Coulomb energy equation is a useful component of binding energy equations since charge symmetry is required for the nuclear contributions.

The Coulomb energy equations are derived in sect. 2 . The data base and details of the least-squares adjustments are presented in sects. 3 and 4 . The discussion and a summary are contained in sects. 5 and 6.

\section{Coulomb energy equations}

\subsection{GENERAL CONSIDERATIONS}

Classical Coulomb energies of charge distributions $e \rho(\boldsymbol{r})$ can be expressed as

$$
E_{\mathrm{C}}(Z, N)=\frac{1}{2} e \int \rho(\boldsymbol{r}) V(\boldsymbol{r}) \mathrm{d}^{3} r .
$$

Here, $V(r)$ is the electrostatic potential associated with the charge distribution. Coulomb displacement energies between isobaric analog states (IAS) and the respective nuclear ground states

$$
\Delta E_{\mathrm{C}}(Z, N)=\frac{e}{2 T} \int \rho_{\mathrm{exc}}(\boldsymbol{r}) V_{\mathrm{core}}(\boldsymbol{r}) \mathrm{d}^{3} \boldsymbol{r},
$$

however, depend on the potential $V_{\text {core }}$ of the charge distribution but also on the distribution $\rho_{\text {exc }}$ of the $N-Z$ excess neutrons. Thus, Coulomb displacement energies can generally not be obtained from total Coulomb energies and vice versa.

The energies $E_{\mathrm{C}}$ and $\Delta E_{\mathrm{C}}$ for the members of an isospin multiplet can in first-order perturbation theory be written as ${ }^{13-15}$ )

$$
\begin{gathered}
E_{\mathrm{C}}\left(A, T, T_{z}\right)=E_{\mathrm{C}}^{(0)}(A, T)-T_{z} E_{\mathrm{C}}^{(1)}(A, T)+\left(3 T_{z}^{2}-T(T+1)\right) E_{\mathrm{C}}^{(2)}(A, T), \\
\Delta E_{\mathrm{C}}\left(A, T, T_{z}\right)=E_{\mathrm{C}}^{(1)}(A, T)-3\left(2 T_{z}-1\right) E^{(2)}(A, T) .
\end{gathered}
$$

Here, $E_{\mathrm{C}}^{(0)}, E_{\mathrm{C}}^{(1)}$ and $E_{\mathrm{C}}^{(2)}$ are the isoscalar, isovector and isotensor Coulomb energies. These quantities are closely related to the coefficients of the isobaric multiplet mass equation ${ }^{16}$ ).

Analytical expressions for $E_{\mathrm{C}}, \Delta E_{\mathrm{C}}, E_{\mathrm{C}}^{(0)}, E_{\mathrm{C}}^{(1)}$ and $E_{\mathrm{C}}^{(2)}$ will be presented in the following sections. Distributions $\rho(\boldsymbol{r})$ and eqs. (1) and (2) will not be used. Instead, quantities $\omega_{i k}$ will be introduced which represent appropriately averaged electrostatic interaction energies $\left\langle(a, b)\left|e^{2} / r\right|(a, b)\right\rangle$ between pairs of protons $a$ and $b$ in the same or in different major shell regions. 


\subsection{GROUND-STATE COULOMB ENERGIES}

The shell-model equation of Carlson and Talmi ${ }^{8,17}$ ) has been adopted in this work to describe the Coulomb energies of nuclear ground states. It has been derived for protons in a single $j$-shell assuming good proton seniority but will be applied as an approximation to much wider regions of nuclei.

Major shell regions with boundaries at magic proton and neutron numbers are considered $\left(Z_{1}=0, Z_{2}=2, Z_{3}=8, Z_{4}=20, Z_{5}=28, Z_{6}=50, Z_{7}=82\right.$ and $\left.N_{i}=Z_{i}\right)$. For the region $Z_{i} \leqslant Z \leqslant Z_{i+1}$ the equation is written as

$$
\begin{aligned}
E_{\mathrm{C}}(Z, N)= & \Omega_{\overline{i-1} \frac{1}{i-1} \frac{1}{2} Z_{i}\left(Z_{i}-1\right)+\Omega_{i-1}{ }_{i} Z_{i}\left(Z-Z_{i}\right)} \\
& +\Omega_{i i} \frac{1}{2}\left(Z-Z_{i}\right)\left(Z-Z_{i}-1\right)+\Pi_{i}\left[\frac{1}{2}\left(Z-Z_{i}\right)\right],
\end{aligned}
$$

where the various terms describe the interactions between pairs of core-core, core-valence and valence-valence protons. Thus, $\Omega_{\overline{i-1} \overline{i-1}}$ (or $\Omega_{\mathrm{cc}}$ ), $\Omega_{\overline{i-1} i}$ (or $\Omega_{\mathrm{cv}}$ ), and $\Omega_{i i}$ (or $\Omega_{\mathrm{vv}}$ ) represent averaged two-body matrix elements involving protons in the core $(\overline{i-1})$ and/or the valence shell $(i)$, respectively. The pairing quantity $\Pi_{i} \equiv \Pi_{i i}\left(\right.$ or $\left.\Pi_{\mathrm{vv}}\right)$ accounts for the increased energy of $(J=0)$-coupled pairs of valence protons.

The interaction energies which depend on the shells and on the number of protons and neutrons within the shell will be expressed as $\Omega_{\overline{i-1} \overline{i-1}}(Z, N)=S(Z, N) \omega_{\overline{i-1} \overline{i-1}}$, $\Omega_{\overline{i-1} i}(Z, N)=S(Z, N) \omega_{\bar{i}-1}, \quad \Omega_{i i}(Z, N)=S(Z, N) \omega_{i i}$ and $\Pi_{i}(Z, N)=S(Z, N) \pi_{i}$. Here, $S(Z, N)$ is a common size factor which accounts for the variation with $Z$ and $N$ of the interaction energies $\Omega$ and $I I$ on account of the change in radius. It will be discussed in the next section.

\subsection{SIZE FACTORS $S(Z, N)$}

Nuclear radii increase with mass number approximately proportional to $A^{1 / 3}$. The matrix elements $\Omega$ are therefore expected to decrease proportionally to $A^{-1 / 3}$ even within a major shell region. However, it was observed earlier ${ }^{18}$ ) that the decrcase is usually weaker and can for "diagonal" regions with valence protons and neutrons in equivalent orbits phenomenologically be well described by $\left(A_{i} / A\right)^{\sigma_{i} / 3}$. Here, $A=Z+N, A_{i}=Z_{i}+N_{i}$ and $\sigma_{i}<1$ (usually). It is not obvious how this equation should be extrapolated into "non-diagonal" regions with valence protons and neutrons in different orbits. Preliminary attempts were discussed earlier ${ }^{19}$ ). The analysis described below showed that expressions which in neutronrich regions give the same isotope shift as in the diagonal region with the same number of neutrons yield very good agreement with the data.

Defining

$$
S_{i} \equiv S\left(Z_{i}, N_{i}\right), \quad \text { with } S_{2}=1,
$$


and shell regions $(i, k)$ by

$$
Z_{i} \leqslant Z \leqslant Z_{i+1}, \quad N_{k} \leqslant N \leqslant N_{k+1},
$$

we introduce the size factors for diagonal and neutron-rich regions,

$(i, i): \quad S(Z, N)=S_{i}\left(\frac{Z_{i}+N_{i}}{Z+N}\right)^{\sigma_{i} / 3}$,

$(i, i+1): \quad S(Z, N)=S_{i}\left(\frac{Z_{i}+N_{i}}{Z+N_{i+1}}\right)^{\sigma_{i} / 3}\left(\frac{Z+N_{i+1}}{Z+N}\right)^{\sigma_{i+1} / 3}$,

$(i, i+2): \quad S(Z, N)=S_{i}\left(\frac{Z_{i}+N_{i}}{Z+N_{i+1}}\right)^{\sigma_{i} / 3}\left(\frac{Z+N_{i+1}}{Z+N_{i+2}}\right)^{\sigma_{i+1} / 3}\left(\frac{Z+N_{i+2}}{Z+N}\right)^{\sigma_{i+2} / 3}$,

and for proton-rich regions based on $S(Z, N)=S(N, Z)$,

$(i+1, i): \quad S(Z, N)=S_{i}\left(\frac{Z_{i}+N_{i}}{Z_{i+1}+N}\right)^{\sigma_{i} / 3}\left(\frac{Z_{i+1}+N}{Z+N}\right)^{\sigma_{i+1} / 3}$,

$(i+2, i): \quad S(Z, N)=S_{i}\left(\frac{Z_{i}+N_{i}}{Z_{i+1}+N}\right)^{\sigma_{i} / 3}\left(\frac{Z_{i+1}+N}{Z_{i+2}+N}\right)^{\sigma_{i+1} / 3}\left(\frac{Z_{i+2}+N}{Z+N}\right)^{\sigma_{i+2} / 3}$.

Eqs. (8) to (12) are schematically displayed in fig. 1. Each arrow represents a factor in the equations. The size factors change continuously at shell crossings.

Eqs. (6) and (8) give

$$
S_{i+1}=S_{i}\left(\frac{A_{i}}{A_{i+1}}\right)^{\sigma_{i} / 3}
$$

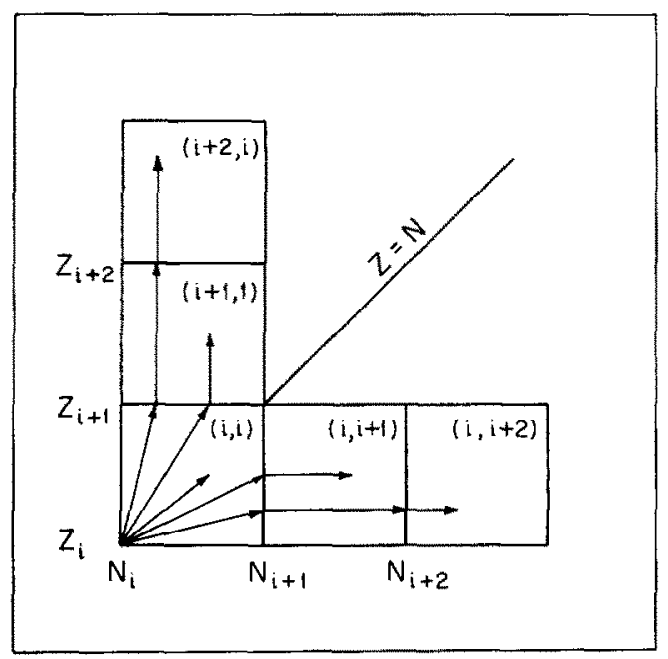

Fig. 1. Schematic representation of the size-factor equations (8) to (12). Each arrow represents a factor in these expressions. 
The isotope and isotone shift coefficients of the size factor are defined by

$$
\begin{aligned}
\gamma_{N} & \equiv \frac{3 A}{S} \frac{\partial S}{\partial N}, \\
\gamma_{Z} & \equiv \frac{3 A}{S} \frac{\partial S}{\partial Z} .
\end{aligned}
$$

The size factor defined in eqs. (6)-(12) has the interesting property that the shift coefficients are exactly or approximately constant for the various regions. We have.

$(i, i)$ :

$(i, i+1)$ :

$(i, i+2)$ :

$(i+1, i)$ :

$(i+2, i)$ :

$$
\begin{array}{ll}
\gamma_{N}=-\sigma_{i}, & \gamma_{Z}=-\sigma_{i}, \\
\gamma_{N}=-\sigma_{i+1}, & \gamma_{Z} \approx-\sigma_{i}, \\
\gamma_{N}=-\sigma_{i+2}, & \gamma_{Z} \approx-\sigma_{i}, \\
\gamma_{N} \approx-\sigma_{i}, & \gamma_{Z}=-\sigma_{i+1}, \\
\gamma_{N} \approx-\sigma_{i}, & \gamma_{Z}=-\sigma_{i+2} .
\end{array}
$$

The corrections in the four equations which are only approximately satisfied are very small and on the order of $\Delta \sigma \Delta Z / A$ or $\Delta \sigma \Delta N / A$.

An alternate possibility to define size factors for off-diagonal regions consists of reversing in eqs. (9) to (12) the sequence of accounting for the number of particles and holes. Eq. (9), for example, can be written as

$$
(i, i+1): \quad S(Z, N)=S_{i+1}\left(\frac{Z_{i+1}+N_{i+1}}{Z_{i+1}+N_{i+1}-H}\right)^{\sigma_{i} / 3}\left(\frac{Z_{i+1}+N_{i+1}-H}{Z_{i+1}+N_{i+1}-H+P}\right)^{\sigma_{i+1} / 3},
$$

whereas the alternate expression is

$$
(i, i+1): \quad S(Z, N)=S_{i+1}\left(\frac{Z_{i+1}+N_{i+1}}{Z_{i+1}+N_{i+1}+P}\right)^{\sigma_{i+1} / 3}\left(\frac{Z_{i+1}+N_{i+1}+P}{Z_{i+1}+N_{i+1}+P-H}\right)^{\sigma_{i} / 3}
$$

However, the two procedures are practically equivalent and calculated Coulomb energies or Coulomb energy differences differ very little.

It is worth noting that eqs. (8) to (12) give equal size factors for the most neutron-rich and proton-rich members of an isospin multiplet. Since the members of an isospin multiplet have the same shell occupation numbers, they will be assigned equal size factors.

\section{4. $\Delta E_{\mathrm{C}}, E_{\mathrm{C}}^{(0)}, E_{\mathrm{C}}^{(1)}$ AND $E_{\mathrm{C}}^{(2)}$ FOR DIAGONAL REGIONS}

Eq. (5) for nuclear ground states describes the energies of the members of an isospin multiplet only approximately. While its major terms are quadratic in $T_{z}$, the pairing term is not and therefore does not satisfy eq. (3). 
Two theoretical approaches have been discussed by Hecht ${ }^{9,10}$ ) for nuclei with valence protons and neutrons in equivalent orbits using isospin $T$ as a good quantum number. They make use of the seniority ${ }^{9}$ ) and the supermultiplet ${ }^{10}$ ) coupling schemes. Numerical analyses using these equations for nuclei in the $1 \mathrm{p}, 1 \mathrm{~d} 2 \mathrm{~s}$, and $1 f_{7 / 2}$ shells have been reported ${ }^{18}$ ). Hecht's supermultiplet equations [eqs. (8.77) to $(8.85)$ in ref. $\left.{ }^{18}\right)$ ] are used in the present work which includes light and heavy nuclei. In the notation of eq. (5) with $\Omega_{i j}=S(Z, N) \omega_{i j}$ and $\Pi_{i}=S(Z, N) \pi_{i}, \Delta E_{\mathrm{C}}$, $E_{\mathrm{C}}^{(0)}, E_{\mathrm{C}}^{(1)}$, and $E_{\mathrm{C}}^{(2)}$ can be expressed as

$$
\Delta E_{\mathrm{C}}(Z, N)=S(Z, N)\left\{\omega_{\bar{i}-1} Z_{i}+\omega_{i i}\left(Z-Z_{i}\right)+\pi_{i}\left[\frac{1}{2} \delta_{\mathrm{oe}} / T+\frac{1}{2} \delta_{\mathrm{oo}}+\frac{1}{2} \delta_{\mathrm{oo}}\left(\alpha^{2}-\frac{1}{3} \beta^{2}\right)\right]\right\} .
$$

Here, $\delta_{\mathrm{oe}}=1$ for $Z=$ odd, $N=$ even, and zero otherwise. The quantities $\delta_{\mathrm{eo}}, \delta_{\mathrm{oo}}$ and $\delta_{\mathrm{ee}}$ are defined accordingly. Also, the ground states of odd-odd nuclei are written as $\mid$ g.s. $\rangle=\alpha|S=0\rangle+\beta|S=1\rangle$. We have $\beta^{2}=3 \alpha^{2}$ if equal population of substates is assumed, and the last term in eq. (17) cancels. In addition

$$
\begin{aligned}
E_{\mathrm{C}}^{(0)}(A, T)= & S\left(\frac{1}{2} A-T, \frac{1}{2} A+T\right)\left\{\frac{1}{8} \omega_{i-1} \frac{1}{i-1} A_{i}\left(A_{i}-2\right)+\frac{1}{4} \omega_{\overline{i-1}}, A_{i}\left(A-A_{i}\right)\right. \\
& +\frac{1}{8} \omega_{i i}\left(A-A_{i}\right)\left(A-A_{i}-2\right)+\frac{1}{4} \pi_{i}\left(A-A_{i}\right)+\frac{1}{6} \omega_{i i} T(T+1) \\
& \left.-\frac{1}{6} \pi_{i}\left(\left(\delta_{\mathrm{oe}}+\delta_{\mathrm{eo}}\right)(T+1)+\delta_{\mathrm{ee}} T+3 \delta_{\mathrm{oo}}-\delta_{\mathrm{oo}}\left(\alpha^{2}-\frac{1}{3} \beta^{2}\right)\right)\right\} \\
E_{\mathrm{C}}^{(1)}(A, T)= & S\left(\frac{1}{2} A-T, \frac{1}{2} A+T\right)\left\{\frac{1}{2} \omega_{\overline{i-1}} A_{i}+\frac{1}{2} \omega_{i l}\left(A-A_{i}-1\right)\right. \\
& \left.+\frac{1}{2} \pi_{i}+\frac{1}{4}\left(\pi_{i} / T\right)\left(\delta_{\mathrm{oe}}-\delta_{\mathrm{eo}}\right)\right\}, \\
E_{\mathrm{C}}^{(2)}(A, T)= & S\left(\frac{1}{2} A-T, \frac{1}{2} A+T\right) \frac{1}{6}\left\{\omega_{i i}+\pi_{i}\left[\delta_{\mathrm{ee}} /(2 T-1)+\left(\delta_{\mathrm{oe}}+\delta_{\mathrm{eo}}\right) / 2 T\right.\right. \\
& \left.\left.-\left(\delta_{\mathrm{oo}} /(2 T-1)\right)\left(\alpha^{2}-\frac{1}{3} \beta^{2}\right)\right]\right\} .
\end{aligned}
$$

\section{5. $\Delta E_{\mathrm{C}} E_{\mathrm{C}}^{(0)}, E_{\mathrm{C}}^{(1)}$ AND $E_{\mathrm{C}}^{(2)}$ FOR OFF-DIAGONAL REGIONS}

Expressions similar to eqs. (18) to (20) for off-diagonal shell regions with valence protons and neutrons in different orbits can be obtained by introducing a particlehole formalism ${ }^{19.20}$ ). Shell-model formulas will be derived in this section for nuclei in off-diagonal regions where neutrons fill a major shell above the proton shell. The extension to the general case is straightforward.

Fig. 2 displays schematically the wave functions of the ground state of a neutronrich nucleus and of its isobaric analog state. The latter is derived by applying the isospin-lowering operator $T^{-}$. The number of particles (neutrons), $P$, and holes (protons), $H$, can be written as

$$
\begin{aligned}
& P=T+\frac{1}{2}\left(A-A_{i+1}\right), \\
& H=T-\frac{1}{2}\left(A-A_{i+1}\right),
\end{aligned}
$$




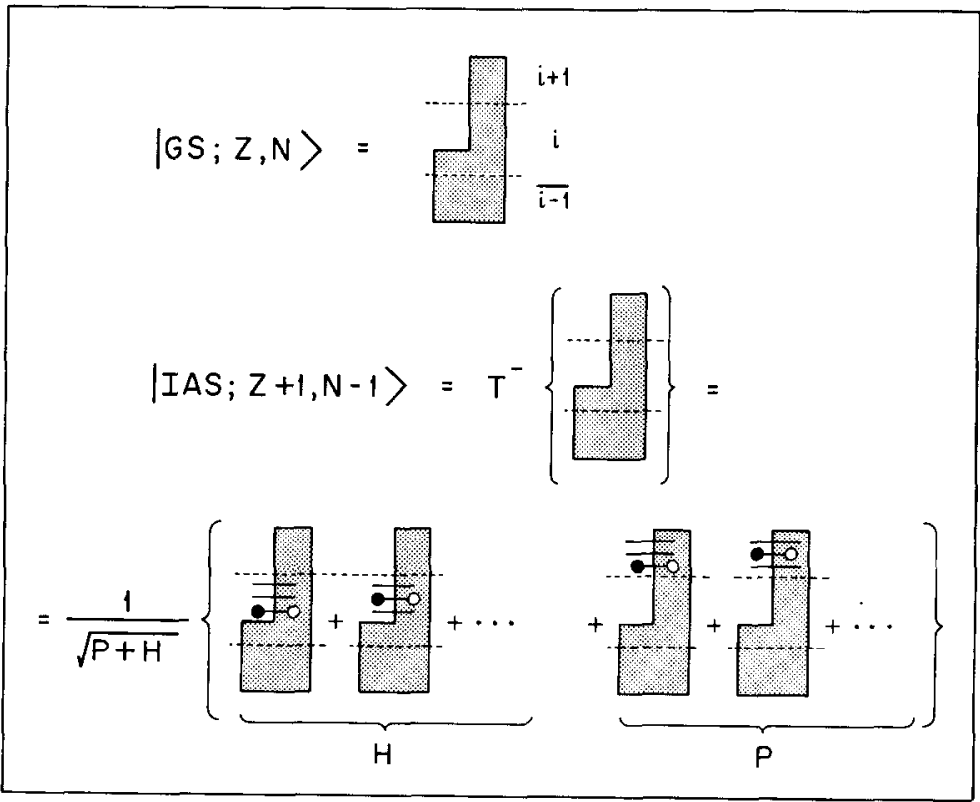

Fig. 2. Schematic representation of the wave functions for ground states and isobaric analog states (IAS) of nuclei with protons and neutrons in different major shell regions.

with

$$
\begin{aligned}
& P+H=2 T, \\
& P-H=A-A_{i+1} .
\end{aligned}
$$

Introducing the appropriate interaction energies $\omega$ and $\pi$, the Coulomb displacement energies can be expressed as

$$
\begin{aligned}
\Delta E_{\mathrm{C}}(Z, N)= & S(Z, N)\left\{\frac{H}{P+H}\left(\omega_{\overline{i-1}} Z_{i}+\omega_{i i}\left(Z-Z_{i}\right)+\frac{1}{H} \pi_{i} \delta_{H}\right)\right. \\
& \left.+\frac{P}{P+H}\left(\omega_{i-1}{ }_{i+1} Z_{i}+\omega_{i+1}\left(Z-Z_{i}\right)\right)\right\} .
\end{aligned}
$$

Here, $\delta_{H}=1$ for $H=$ odd, and zero otherwise. Eq. (23) can be simplified by introducing the identity

$$
\omega_{i-1}{ }_{i+1} Z_{i}+\omega_{i+1}\left(Z_{i+1}-Z_{i}\right)=\omega_{\bar{i} i+1} Z_{i+1},
$$

and the difference

$$
\Delta \omega \equiv \omega_{\bar{i} i+1}-\omega_{i+1},
$$


which is presumably very small. Eq. (23) becomes

$$
\begin{aligned}
\Delta E_{\mathrm{C}}(Z, N)= & S(Z, N)\left\{\frac{H}{P+H}\left(\omega_{\overline{i-1}} Z_{i}+\omega_{i i}\left(Z-Z_{i}\right)+\frac{1}{H} \pi_{i} \delta_{H}\right)\right. \\
& \left.+\frac{P}{P+H} \omega_{\bar{i} i+1} Z+\frac{P H}{P+H} \Delta \omega\right\} .
\end{aligned}
$$

Combining eqs. (26) and (3) with eq. (5) applied to the ground states of the most neutron-rich and proton-rich members of an isospin multiplet makes it possible to derive expression for the scalar, vector and tensor Coulomb energies. The result is

$$
\begin{aligned}
& E_{\mathrm{C}}^{(0)}\left(A_{i+1}+P-H, \frac{1}{2}(P+H)\right) \\
& =S\left(\frac{1}{2} A_{i+1}-H, \frac{1}{2} A_{i+1}+P\right)\left\{\frac{1}{8} \omega_{\overline{i-1} \overline{i-1}} A_{i}\left(A_{i}-2\right)+\frac{1}{4} \omega_{\overline{i-1}} A_{i}\left(A_{i+1}-A_{i}\right)\right. \\
& +\frac{1}{8} \omega_{i i}\left(A_{i+1}-A_{i}\right)\left(A_{i+1}-A_{i}-2\right)+\frac{1}{4} \pi_{i}\left(A_{i+1}-A_{i}\right) \\
& -\frac{1}{4} \omega_{\overline{i-1}}{ }_{i} A_{i} H+\frac{1}{4} \omega_{\bar{i}}{ }_{i+1} A_{i+1} P \\
& -\frac{1}{4} \omega_{i i}\left(A_{i+1}-A_{i}\right) H+\frac{1}{6} \omega_{i i} H(H+2)-\frac{1}{6}\left(\omega_{i i+1}-\Delta \omega\right) P H+\frac{1}{6} \omega_{i+1}{ }_{i+1} P(P-1) \\
& \left.-\frac{1}{6} \pi_{i}\left(2 H+\delta_{H}\right)+\frac{1}{6} \pi_{i+1}\left(P-\delta_{P}\right)\right\}, \\
& E_{\mathrm{C}}^{(1)}\left(A_{i+1}+P-H, \frac{1}{2}(P+H)\right) \\
& =S\left(\frac{1}{2} A_{i+1}-H, \frac{1}{2} A_{i+1}+P\right) \frac{1}{P+H}\left\{\frac{1}{2} \omega_{i-1} A_{i} H+\frac{1}{2} \omega_{i+1}^{-} A_{i+1} P\right. \\
& +\frac{1}{2} \omega_{i i}\left(A_{i+1}-A_{i}\right) H-\frac{1}{2} \omega_{i i} H(H+1) \\
& \left.+\frac{1}{2} \omega_{i+1 i+1} P(P-1)+\frac{1}{2} \pi_{i}\left(H+\delta_{H}\right)+\frac{1}{2} \pi_{i+1}\left(P-\delta_{P}\right)\right\}, \\
& E_{C}^{(2)}\left(A_{i+1}+P-H, \frac{1}{2}(P+H)\right) \\
& =S\left(\frac{1}{2} A_{i+1}-H, \frac{1}{2} A_{i+1}+P\right) \\
& \omega_{i j} H(H-1)+\pi_{i}\left(H-\delta_{H}\right)+2\left(\omega_{\tilde{i} i+1}-\Delta \omega\right) P H \\
& \times \frac{+\omega_{i+1 i+1} P(P-1)+\pi_{i+1}\left(P-\delta_{P}\right)}{6(P+H)(P+H-1)} .
\end{aligned}
$$

Here, $\delta_{H}=1$ or 0 for $H=$ odd or even, and similarly for $\delta_{P}$. In heavy nuclei eq. (3) with eqs. (27), (28) and (29) should be applied preferably to the neutron-rich members of an isospin multiplet where it is expected to give better agreement with the data.

\section{Data base}

Most of the Coulomb displacement energy data used in the present work were taken from the compilation of Courtney and Fox ${ }^{21}$ ). Only displacement energies 
for neutron-rich ground states (or low excited states with $E_{\mathrm{x}}<500 \mathrm{keV}$ if not available) were included and uncertainties were required not to exceed $60 \mathrm{keV}$. The displacement energies between the ground states of mirror nuclei with $T=\frac{1}{2}$ were replaced by more precise values from a recent mass evaluation ${ }^{22}$ ). Additional data for the $\mathrm{Sn}$ and $\mathrm{Te}$ isotopes ${ }^{23}$ ) as well as over 25 recently measured displacement energies for rare-earth nuclei ${ }^{6,7}$ ) were also included. Whereas only data for neutronrich nuclei were considered in the least-squares adjustments, certain proton-rich data were used for comparison.

\section{Numerical analysis and results}

A least-squares minimization has been performed to describe the Coulomb displacement energies of the preceding chapter by the shell-model equations for diagonal and off-diagonal regions, eqs. (17) and (26). The interaction energies $\omega$ and $\pi$ together with the exponents $\sigma$ of the size factors, eqs. (8) to (13), are used as adjustable parameters. The problem is non-linear as it contains the dependence on the exponent $\sigma$. A computer program ${ }^{24}$ ) was employed. It determines the minimum of the sum of squares of $m$ functions in $n$ variables using a finite difference algorithm.

Three different procedures have been used which will be denoted (A) diagonal, (B) global, and (C) ground-state procedure, respectively.

$(A)$ Diagonal procedure: In this procedure the parameters $\omega$ and $\pi$ are obtained individually for each diagonal region, eq. (17). They are subsequently used, eq. (26), to predict the displacement energies for the off-diagonal regions. The interaction energies $\omega_{\overline{i-1}} \overline{i-1}$ in $\Omega_{\overline{i-1}} \overline{i-1}=S(Z, N) \omega_{\overline{i-1}} \overline{i-1}$ are not included in the optimizations. Instead, a value $\Omega_{\Gamma_{1}}=\Omega_{11}=876 \mathrm{keV}$ was deduced for $Z=N=2$ from the Coulomb displacement energy between ${ }^{3} \mathrm{He}$ and ${ }^{3} \mathrm{H}$, corrected for the different experimental charge radii of ${ }^{3} \mathrm{He}$ and ${ }^{4} \mathrm{He}$. In the higher diagonal regions, the interaction energy $\Omega_{\bar{i} i}$ between core-core protons can be expressed as an appropriate sum over the interaction energies $\Omega_{\overline{i-1} \overline{i-1}}, \Omega_{\overline{i-1} i}, \Omega_{i i}$ and $\Pi_{i}$. This is due to the fact that $E_{\mathrm{C}}\left(Z_{i+1}, N_{i+1}\right)$ can be written with eq. (5) in two different ways involving two different shell regions. The result for $Z=N=\frac{1}{2} A_{i+1}$ is

$$
\begin{aligned}
\Omega_{\bar{i} \frac{1}{2}} Z_{i+1}\left(Z_{i+1}-1\right)= & \Omega_{\overline{i-1}} \frac{1}{i-1} Z_{i}\left(Z_{i}-1\right)+\Omega_{\overline{i-1}} Z_{i}\left(Z_{i+1}-Z_{i}\right) \\
& +\Omega_{i i} \frac{1}{2}\left(Z_{i+1}-Z_{i}\right)\left(Z_{i+1}-Z_{i}-1\right)+\Pi_{i} \frac{1}{2}\left(Z_{i+1}-Z_{i}\right),
\end{aligned}
$$

and accordingly for the quantities $\omega$ and $\pi$.

There are four adjustable parameters for each diagonal region, $\omega_{\overline{i-1}} i, \omega_{i i}, \pi_{i}$ and $\sigma_{i}$. In preliminary tests it was found that the values of the pairing quantity $\pi_{i}$ decrease rapidly with increasing $i$ becoming practically zero for $i>4$. Thus, $\pi_{i}$ was set equal to zero for the upper three regions reducing the number of adjustable parameters in these regions to three. Due to the scarcity of data for very heavy 
TABLE 1

Average matrix elements $\Omega_{i j}=S_{i} \omega_{i l}$ and $\Pi_{i}=S_{i} \pi_{i}$ for the Coulomb interactions between core-core, core-valence, and valence-valence protons in major shell regions (the size factors $S_{i} \equiv S\left(Z_{i}, N_{i}\right)$ are calculated for the lightest nucleus in each diagonal region)

\begin{tabular}{|c|c|c|c|c|c|}
\hline$Z_{i}$ & $\sigma$ & $\begin{array}{c}\Omega_{\text {cc }} \\
(\mathrm{keV})\end{array}$ & $\begin{array}{c}\Omega_{\mathrm{cv}} \\
(\mathrm{keV})\end{array}$ & $\begin{array}{c}\Omega_{\mathrm{vv}} \\
(\mathrm{keV})\end{array}$ & $\begin{array}{c}\Pi_{\mathrm{vv}} \\
(\mathrm{keV})\end{array}$ \\
\hline \multicolumn{6}{|c|}{ procedure $(A)$ : diagonal } \\
\hline 2 & 0.50020 & 876.000 & 455.627 & 666.930 & 264.5 \\
\hline 8 & 0.86236 & 485.844 & 461.126 & 519.222 & 118.1 \\
\hline 20 & 0.21455 & 375.520 & 363.714 & 329.350 & 76.0 \\
\hline 28 & 0.92477 & 359.159 & 343.989 & 350.166 & 0.0 \\
\hline 50 & 0.86718 & 292.577 & 289.106 & 281.612 & 0.0 \\
\hline 82 & 1.41978 & 250.724 & 257.145 & 276.391 & 0.0 \\
\hline \multicolumn{6}{|c|}{ procedure $(B):$ global } \\
\hline 2 & 0.42829 & 876.000 & 458.100 & 628.483 & 283.1 \\
\hline 8 & 0.93554 & 487.860 & 460.840 & 536.841 & 118.8 \\
\hline 20 & 0.25890 & 371.951 & 363.506 & 338.727 & 80.6 \\
\hline 28 & 0.70843 & 356.271 & 341.802 & 311.465 & 0.0 \\
\hline 50 & 0.78401 & 296.970 & 287.582 & 269.211 & 0.0 \\
\hline 82 & 0.76568 & 253.338 & 244.848 & 190.851 & 0.0 \\
\hline & $(0.77784)$ & $(253.414)$ & $(244.774)$ & $(227.640)$ & 0.0 \\
\hline \multicolumn{6}{|c|}{ procedure $(C)$ ground states } \\
\hline 2 & 0.64910 & 876.000 & 451.418 & 725.390 & 215.3 \\
\hline 8 & 0.91872 & 471.493 & 460.884 & 532.830 & 100.8 \\
\hline 20 & 0.21986 & 370.581 & 363.435 & 331.536 & 50.3 \\
\hline 28 & 0.92477 & 356.300 & 343.989 & 350.166 & 0.0 \\
\hline 50 & 0.86718 & 291.839 & 289.106 & 281.612 & 0.0 \\
\hline 82 & 1.41978 & 250.488 & 257.145 & 276.391 & 0.0 \\
\hline
\end{tabular}

The quoted digits have no physical significance but are included only to facilitate numerical calculations.

nuclei, $\sigma_{8}=\sigma_{7}$ was assumed. This effectively removes the influence from the magic number $N_{8}=126$. Odd-odd nuclei were excluded from the least-squares adjustment. Equal weight factors were used.

The parameters $\omega$ and $\pi$ from procedure (A) are listed in table 1. The mean values $\langle\varepsilon\rangle$ and standard deviations $\sigma_{\varepsilon}$ for the differences between the experimental and calculated Coulomb energies are shown in table 2 for all diagonal and the extrapolated off-diagonal regions. Here, odd-odd nuclei are included assuming $\alpha^{2}-\frac{1}{3} \beta^{2}=0$. Furthermore, eq. (26) for the off-diagonal regions is used with $\Delta \omega=0$. The individual differences $\varepsilon$ between the experimental and calculated energies are displayed in fig. 3 . The symbols which are used group the differences according to their positive or negative deviations from zero.

$(B)$ Global procedure: This procedure is very similar to procedure (A) except that the least-squares adjustment of the 21 parameters $\omega, \pi$ and $\sigma$ was carried out simultaneously for all diagonal and off-diagonal regions. Odd-odd nuclei were 
TABLE 2

Mean values $\langle\varepsilon\rangle$ and standard deviations $\sigma_{\varepsilon}$ for the differences $\varepsilon$ between experimental and calculated Coulomb displacement energies for diagonal and off-diagonal shell regions with $N>Z$ including the mirror nuclei with $T=\frac{1}{2}$; the three procedures are explained in the text

\begin{tabular}{|c|c|c|c|c|c|c|c|}
\hline \multirow{2}{*}{ Region } & \multirow{2}{*}{$\begin{array}{l}\text { Number } \\
\text { of cases }\end{array}$} & \multicolumn{2}{|c|}{ Procedure (A) } & \multicolumn{2}{|c|}{ Procedure (B) } & \multicolumn{2}{|c|}{ Procedure (C) } \\
\hline & & $\begin{array}{c}\langle\varepsilon\rangle \\
(\mathrm{keV})\end{array}$ & $\begin{array}{c}\sigma_{\varepsilon} \\
(\mathrm{keV})\end{array}$ & $\begin{array}{c}\langle\varepsilon\rangle \\
(\mathrm{keV})\end{array}$ & $\begin{array}{c}\sigma_{\varepsilon} \\
(\mathrm{keV})\end{array}$ & $\begin{array}{c}\langle\varepsilon\rangle \\
(\mathrm{keV})\end{array}$ & $\begin{array}{c}\sigma_{\varepsilon} \\
(\mathrm{keV})\end{array}$ \\
\hline$(2,2)$ & 14 & -9.3 & 82.9 & 2.6 & 79.6 & -18.6 & 96.6 \\
\hline$(3,3)$ & 39 & 5.0 & 50.5 & 11.3 & 49.1 & 0.0 & 46.5 \\
\hline$(4,4)$ & 33 & 4.8 & 29.7 & 7.4 & 30.7 & 3.8 & 31.6 \\
\hline$(5,5)$ & 33 & -0.7 & 41.0 & 3.4 & 52.2 & -0.7 & 41,0 \\
\hline$(6,6)$ & 29 & 1.7 & 18.2 & 10.3 & 22.0 & 1.7 & 18.2 \\
\hline$(7,7)$ & 8 & 0.0 & 43.1 & -21.5 & 51.9 & 0.0 & 43.1 \\
\hline diagonal & 156 & 1.6 & 42.4 & 6.2 & 45,9 & -0.7 & 43.7 \\
\hline$(2,3)$ & 4 & -95.0 & 68.9 & -68.7 & 113.5 & -245.5 & 74.5 \\
\hline$(3,4)$ & 10 & -34.1 & 27.5 & -22.2 & 32.9 & 150.4 & 74.0 \\
\hline$(4,5)$ & 20 & 10.8 & 24.9 & 5.1 & 24.0 & -546.5 & 61.9 \\
\hline$(5,6)$ & 40 & -76.3 & 48.3 & -11.7 & 42.3 & -31.2 & 46.4 \\
\hline$(6,7)$ & 58 & -71.4 & 62.9 & 3.4 & 34.9 & -500.1 & 134.8 \\
\hline off-diagonal & 132 & -58.3 & 60.4 & -5.0 & 39.6 & -308.1 & 272.4 \\
\hline total & 288 & -25.8 & 60.6 & 1.0 & 43.4 & -141.6 & 250.8 \\
\hline
\end{tabular}

excluded in the diagonal regions (only), and $\alpha^{2}-\frac{1}{3} \beta^{2}=0$ and $\Delta \omega=0$ was assumed for the off-diagonal regions.

The parameters $\omega$ and $\pi$ from procedure (B) are included in table 1. Values for $\langle\varepsilon\rangle$ and $\sigma_{\varepsilon}$ (including all odd-odd nuclei) are shown in table 2. The individual differences are displayed in fig. 4 using the same format as fig. 3 .

(C) Ground-state procedure: Procedures (A) and (C) are very similar for the diagonal regions. The parameters $\omega$ and $\pi$ are again obtained from independent least-squares adjustments for the diagonal regions whereas the displacement energies for the off-diagonal regions are obtained by extrapolation. The basic difference between the two procedures is that in (C) instead of eqs. (17) and (26) for $\Delta E_{\mathrm{C}}$, eq. (5) for $E_{C}$ is used in all regions, and $\Delta E_{C}$ is simply obtained by calculating the energy differences between the ground states of neighboring nuclei. This assumption is usually made for the Coulomb energy contributions in atomic mass equations [see e.g. ref. ${ }^{25}$ )]. The size factors eqs. (8) to (13) are again employed. Procedure (C) thus assumes that the Coulomb energies of the isobaric analog states and the ground states in the same nucleus are equal.

The parameters $\omega$ and $\pi$ from procedure (C) are also included in table 1. Similarly, values for $\langle\varepsilon\rangle$ and $\sigma_{\varepsilon}$ are included in table 2 , and the individual differences are displayed in fig. 5 . 


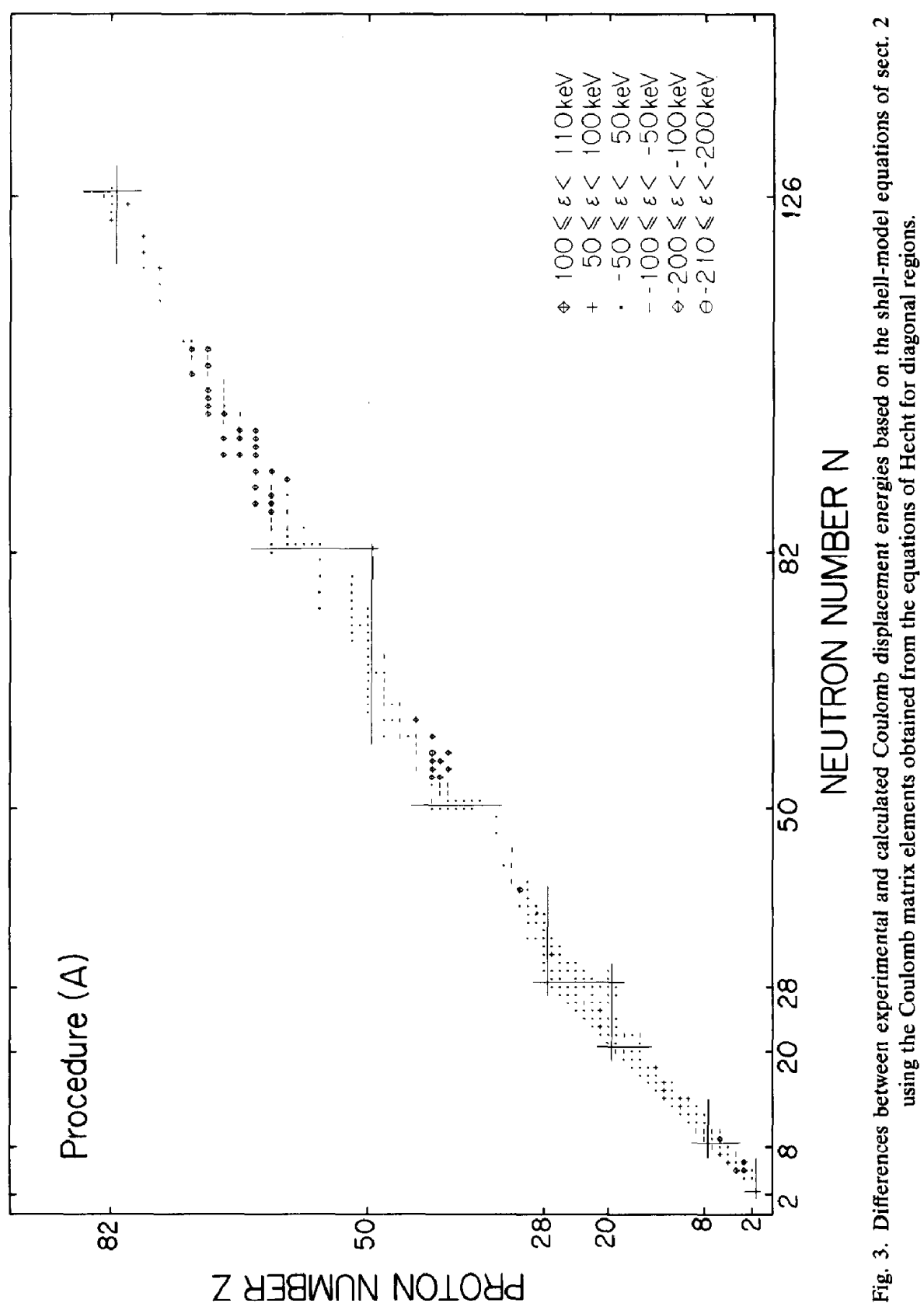




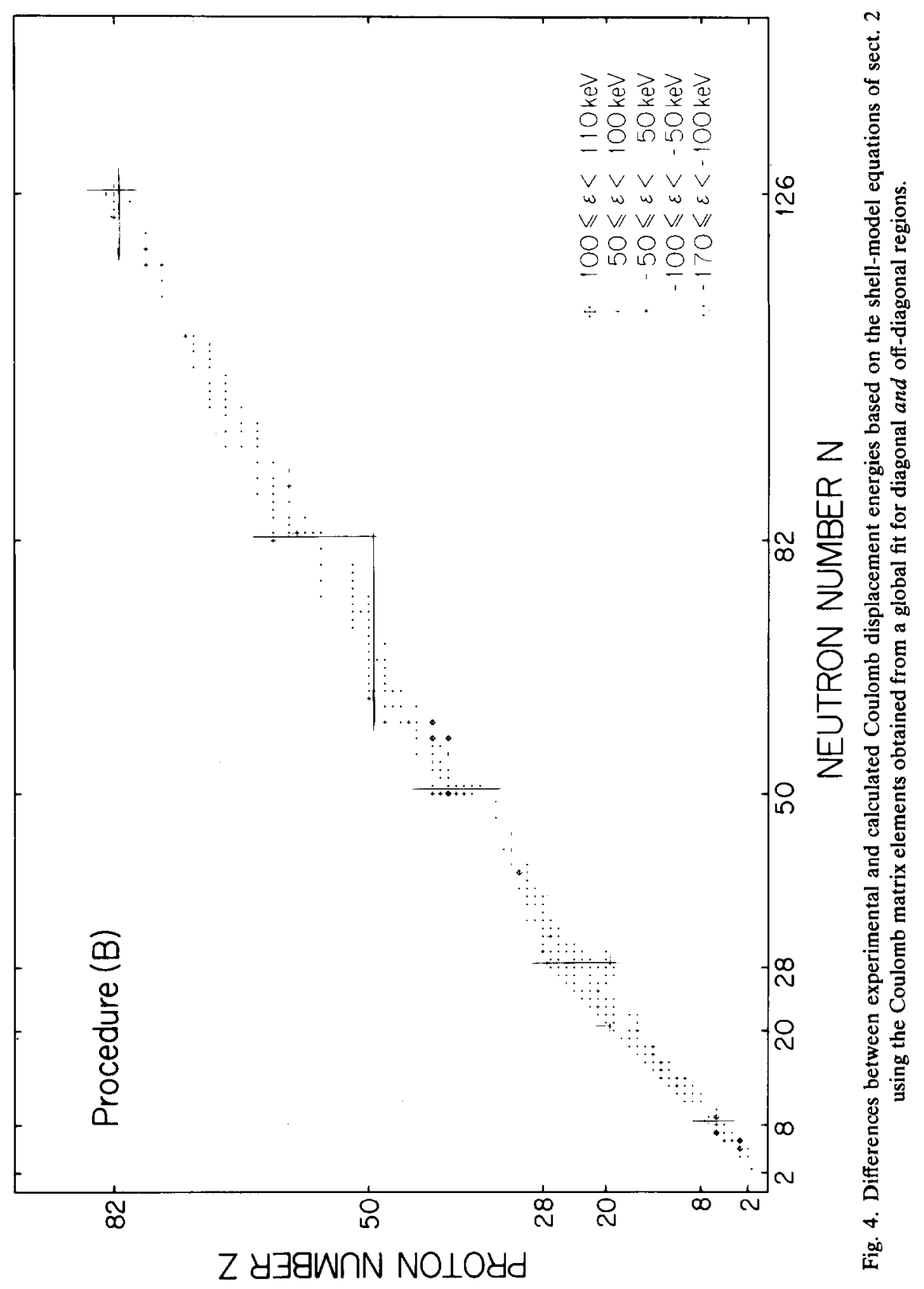




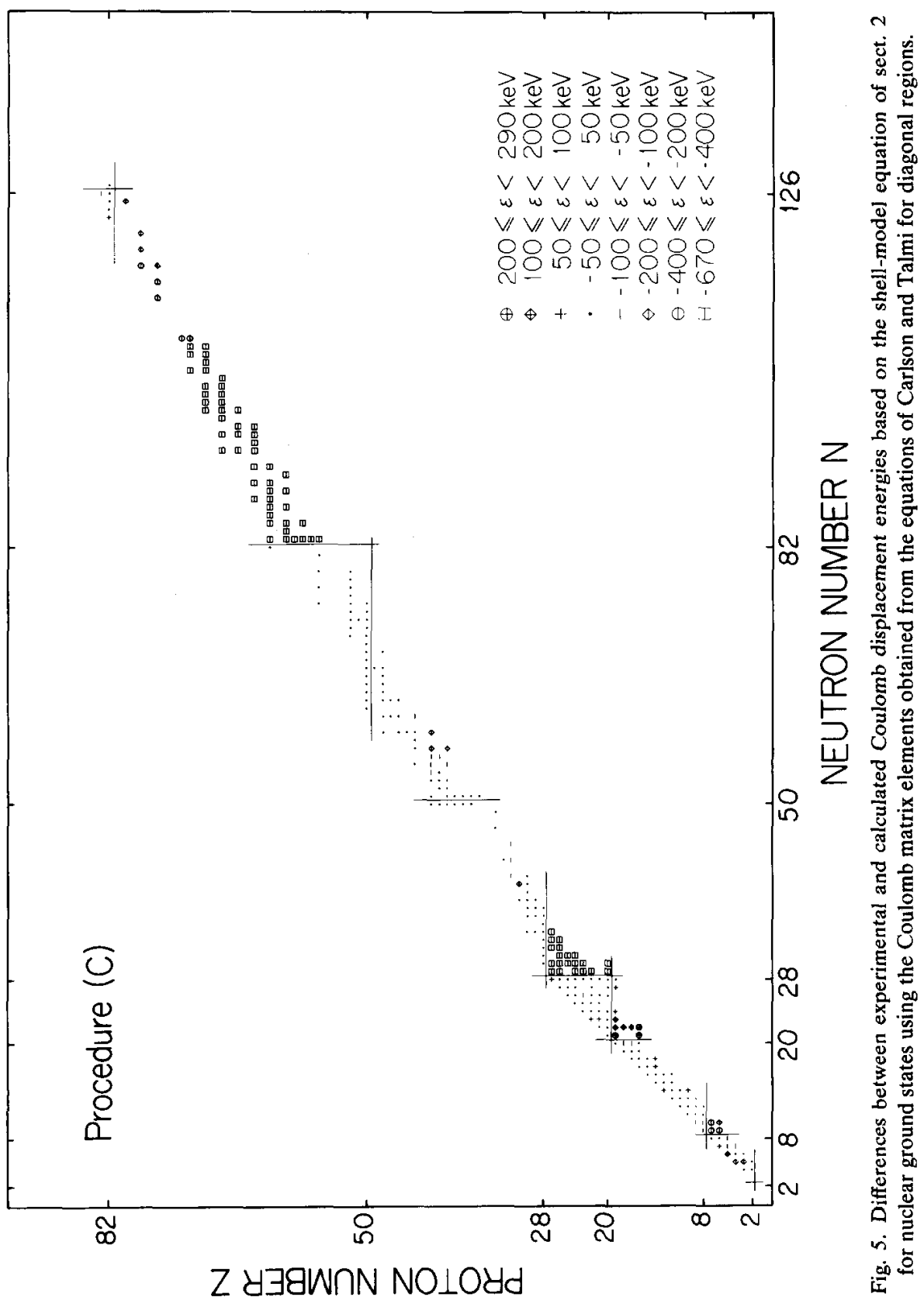


TABLE 3

Mean values $\langle\varepsilon\rangle$ and standard deviations $\sigma_{\varepsilon}$ for the differences between experimental and calculated Coulomb energies of proton-rich nuclei based on the global procedure; see text

\begin{tabular}{lccccccc}
\hline & \multicolumn{3}{c}{ Mirror nuclei $T>\frac{1}{2}$} & & \multicolumn{3}{c}{ Proton-rich IAS } \\
\cline { 2 - 3 } Region & $\begin{array}{c}\text { number } \\
\text { of cases }\end{array}$ & \multicolumn{1}{c}{$\begin{array}{c}\langle\varepsilon\rangle \\
(\mathrm{keV})\end{array}$} & $\begin{array}{c}\sigma_{\varepsilon} \\
(\mathrm{keV})\end{array}$ & & $\begin{array}{c}\text { number } \\
\text { of cases }\end{array}$ & $\begin{array}{c}\langle\varepsilon\rangle \\
(\mathrm{keV})\end{array}$ & $\begin{array}{c}\sigma_{\varepsilon} \\
(\mathrm{keV})\end{array}$ \\
\hline$(2,2)$ & 6 & -51.0 & 122.5 & & 4 & -64.6 & 86.0 \\
$(3,3)$ & 24 & -35.1 & 63.6 & & 14 & -30.9 & 44.6 \\
$(4,4)$ & 3 & 89.6 & 18.2 & & 1 & 113.3 & \\
diagonal & 33 & -26.7 & 81.9 & & 19 & -30.4 & 63.8 \\
$(2,3)$ & 1 & -317.9 & & & 1 & -170.1 & \\
$(3,4)$ & 2 & -86.1 & 27.4 & & 1 & -48.7 & \\
off-diagonal & 3 & -163.4 & 135.2 & & 2 & -109.4 & 85.9 \\
total & 36 & -38.1 & 93.0 & 21 & -37.9 & 67.8 \\
\hline
\end{tabular}

A selected sct of Coulomb energy data for proton-rich nuclei was compared to the predictions using the parameters $\omega$ and $\pi$ from the global procedure (B). The mean values $\langle\varepsilon\rangle$ and standard deviations $\sigma_{\varepsilon}$ for the various regions shown in table 3 are for two types of data: (i) Coulomb energy differences between the ground states of all available higher-order mirror nuclei $\left(T>\frac{1}{2}\right.$ ), (ii) Coulomb displacement encrgies between the two most proton-rich members of an isospin multiplet. Nuclei which are unstable with respect to proton emission were excluded. The data of type (i) were compared to values calculated with eq. (5). The data of type (ii) were compared with eqs. (5) and (17) or (26) according to the identity

$$
\Delta E_{\mathrm{C}}(\mathrm{p}-\text { rich })+\Delta E_{\mathrm{C}}(\mathrm{n}-\text { rich })=\left(E_{\mathrm{C}}(\mathrm{p}-\text { rich })-E_{\mathrm{C}}(\mathrm{n}-\text { rich })\right) / T .
$$

Fig. 6 displays the core-core, core-valence and valence-valence interaction energies $\Omega$ (including size factors) obtained from the global procedure (B) for nuclei along the line of $\beta$-stability. Also included in fig. 6 (dotted line) is an arbitrarily normalized function which decreases as $A^{-1 / 3}$.

\section{Discussion}

The approach taken in the present work to calculate the ground-state Coulomb energies and Coulomb displacement energies involves several important approximations. The first approximation involves the use of a simplified shell model with isospin and of expressions based on first-order perturbation theory. Furthermore, Coulomb displacement energies are known ${ }^{11,12}$ ) to contain contributions from numerous small effects including those from charge-dependent nuclear forces, from 


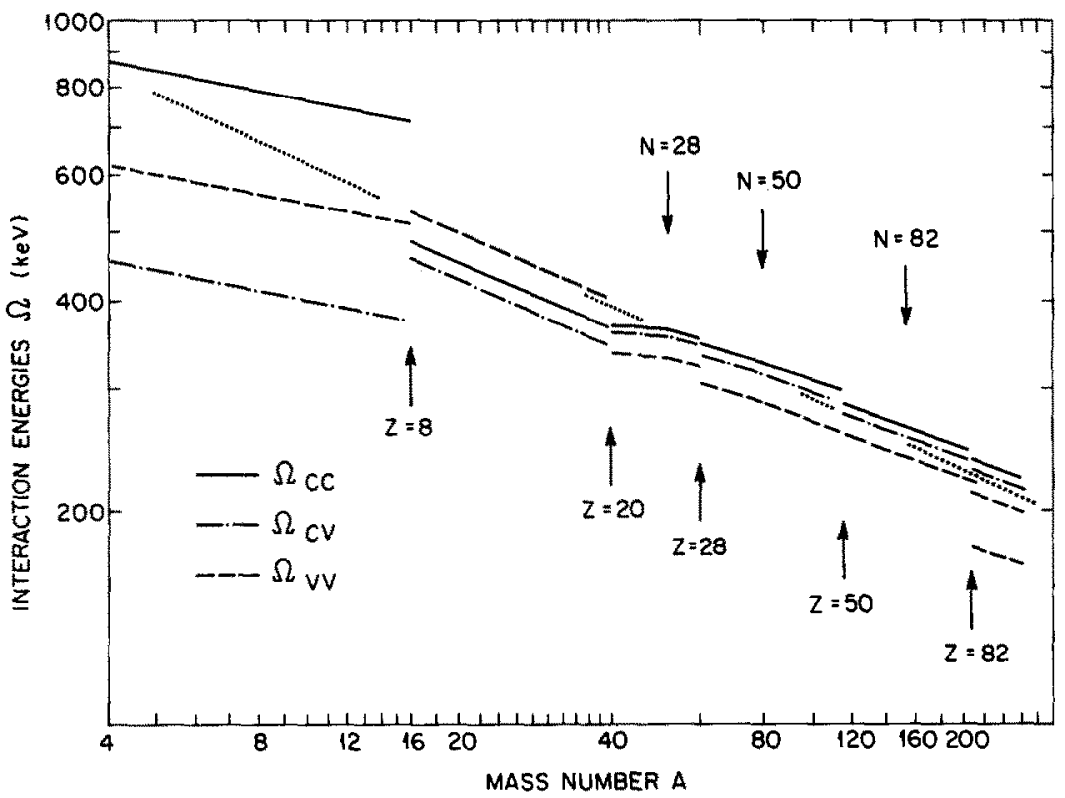

Fig. 6. Coulomb interaction energies $\Omega(Z, N)=S(Z, N)_{\omega}$ for core-core, core-valence, and valencevalence proton pairs for nuclei along the line of $\beta$-stability. The size factor $S(Z, N)$ accounts for the increase in radius. The dotted line is an arbitrarily normalized function decreasing as $A^{-1 / 3}$.

exchange, the electromagnetic spin-orbit interaction, isospin mixing and the Thomas-Ehrman shift. Whereas exchange effects are included in the matrix elements $\Omega$ of sect. 2, none of the other effects are likely to be correctly described by the shell-model equations. The good agreement obtained in sect. 4 suggests that these effects have a relatively smooth dependence on $Z$ and $N$. Therefore, it is reasonable to assume that a considerable part of these effects is included in the parameters $\Omega$ and $\Pi$. Hence, the average combined direct and exchange Coulomb matrix elements $\Omega$ and $I$ of table 1 are likely to contain systematic errors of 3 to $10 \%$, particularly for light nuclei. Another approximation stems from the fact that the major shell regions are not based on a single value of $j$ (except for the $1 \mathbf{f}_{7 / 2}$ shell). Therefore, the shell-model equations are only approximate and the Coulomb interaction energies $\Omega$ and $\Pi$ represent appropriately averaged values. Again, the good agreement which was achieved for the Coulomb displacement energies seems to justify this approach. The approach is analogous to that used by Zeldes ${ }^{26}$ ) to describe nuclear masses on the basis of averaged nuclear interaction energies.

A common underlying assumption used by the semi-empirical mass and the Coulomb energy equations is the assumed smooth variation of the interaction energies (except for the pairing term) within shell regions. Since the nuclear and Coulomb interactions are of short and long range, respectively, and the former is 
stronger than the latter, the agreement between the experimental and calculated values should be considerably better for the Coulomb interaction. The present work shows that this expectation is indeed satisfied.

The three procedures (A), (B) and (C) described in the preceding section lead to different results as is evident from table 2 and figs. 3 to 5 . In the diagonal regions the agreement between the experimental and calculated values is equally good for all three procedures with a standard deviation of about $45 \mathrm{keV}$. In the off-diagonal regions, however, a distinctly different behavior is apparent. In procedure (A) the differences $\varepsilon$ between experimental and calculated values deteriorate gradually in the extrapolation away from the diagonal regions. In procedure $(B)$ the same very good agreement prevails for the diagonal and off-diagonal regions, whereas a discontinuous discrepancy of several hundred $\mathrm{keV}$ becomes apparent at the transition to most off-diagonal regions in procedure $(\mathrm{C})$.

The good agreement for diagonal regions (including odd-odd nuclei) in procedures (A) and (B) reflects upon the appropriateness of the shell-model eq. (17) with the size factor eq. (8). The gradual deterioration in procedure $(A)$ in the off-diagonal regions is an effect quite common in the extrapolation of semi-empirical equations. It is particularly apparent in the off-diagonal regions $(5,6)$ and $(6,7)$ which include data relatively far from diagonal regions. However, the accumulated error is significantly less, $\sim 15 \%$, of that encountered in the cxtrapolation of mass equations ${ }^{27,28}$ ). An attempt was made to explain the negative values of $\varepsilon$ by invoking a finite value for $\Delta \omega$ of eq. (26). However, the corrections introduced appear to be too small and of the wrong sign. Different analytical expressions for the size factors could also not explain the systematic deviations. However, it appears that the deviations which are particularly prominent in the regions $(5,6)$ centered near $A \approx 100$ and $(6,7)$ centered near $A \approx 160$ are of physical origin and can qualitatively be explained by deformation effects. Deformed shapes lead to increased rms radii and hence decreased Coulomb energies. Reductions up to about $150 \mathrm{keV}$ for the most strongly deformed rare-earth nuclei are indeed in agreement with an earlier analysis of this effect ${ }^{6}$ ). No attempts have been made to explicitly include deformation effects by modifying the size factor, for example. Additional parameters would have to be introduced. The good agreement obtained for the strongly deformed nuclei ${ }^{232} \mathrm{Th}$ and ${ }^{238} \mathrm{U}$ is not significant. The scarcity of data in the diagonal region $(7,7)$ made it necessary to include ${ }^{209} \mathrm{~Pb},{ }^{232} \mathrm{Th}$ and ${ }^{238} \mathrm{U}$ in the determination of the respective interaction energies $\omega$.

The good agreement of about $45 \mathrm{keV}$ obtained from procedure (B) over the entire range of nuclei shows that the deformation effects can be approximately accounted for by small changes in the interaction energies $\omega$. Unless deformation effects can be treated more reliably, the parameters from procedure (B) should be used in extrapolations even though the slightly different parameters of procedurc (A) are probably more realistic in the description of Coulomb energies of nondeformed nuclei. 
The good agreement of procedure $(\mathrm{C})$ in diagonal regions is expected as it differs from procedure (A) only in the use of a different small pairing term. The sudden onset of strong discrepancies with the addition of even a single neutron or proton hole in an off-diagonal region (particularly for $N>28, Z<28, N>82$ and $Z<82$ ) points to a significant difference of 400 to over $600 \mathrm{keV}$ between the Coulomb energies in the nuclear ground and isobaric analog states of such nuclei. The reason for this behavior is the fact that the ground state Coulomb energy eq. (5) does not account for the shell occupation of the "excess" proton in the IAS (see fig. 2). Any discrete difference between the Coulomb interaction energies in neighboring major shell regions will lead to different Coulomb energies for states where the protons do not occupy the same major shells. The discontinuities observed in procedure (C) are therefore a manifestation of shell effects.

While the dependence of Coulomb energy differences on nuclear configurations is well established, Coulomb energy equations often assume equal Coulomb energies for ground and isobaric analog states. The above results demonstrate that this is a reasonable assumption for regions where valence protons and neutrons occupy orbits in equivalent major shell regions. However, it is a very poor approximation when they occupy different major shell regions. The result is of particular importance for liquid-drop-model Coulomb energy equations with parameters deduced from experimental Coulomb displacement energies. Such equations are expected to contain systematic errors of typically $500 \mathrm{keV}$ when used to calculate the (unobservable) total Coulomb energies of nuclear ground states in off-diagonal regions. The observed smooth transition of Coulomb displacement energies between diagonal and off-diagonal regions [e.g. fig. 8.13 of ref. ${ }^{18}$ )], on the other hand, is much better represented by such equations and is also in accordance with the smooth transition incorporated in the shell-model eq. (26) for $\Delta E_{C}$ in off-diagonal regions.

The size factors $S(Z, N)$ which are introduced in subsect. 2.3 [see also eqs. (9a) and (9b)] change continuously at shell crossings. Other size factors with this property have also been considered including the approach of ref. $\left.{ }^{19}\right)$ and (for regions $(i, i+1)$ )

$$
\begin{aligned}
S(Z, N) & =S_{i+1}\left(\frac{A_{i+1}}{A_{i+1}-H}\right)^{\sigma_{i} / 3}\left(\frac{A_{i+1}}{A_{i+1}+P}\right)^{\sigma_{i+1} / 3} \\
& =S_{i}\left(\frac{Z_{i}+N_{i}}{Z+N_{i+1}}\right)^{\sigma_{i} / 3}\left(\frac{Z_{i+1}+N_{i+1}}{Z_{i+1}+N}\right)^{\sigma_{i+1} / 3} .
\end{aligned}
$$

Here $P$ and $H$ are again the number of particles and holes. While eq. (32) is somewhat more symmetric in $Z$ and $N$ than eq. (9), the isotope and isotone shift coefficients $\gamma_{N}$ and $\gamma_{Z}$ are not constant. The fact that eqs. (8) to (12) give much better agreement with the data supports the importance of isotope and isotone shift considerations.

Proton-rich nuclei are not included in the determination of the interaction energies. However, table 3 displays the comparison based on the global procedure 
(B) between certain experimental and calculated Coulomb energy differences. Most of the data are for the diagonal regions $(2,2)(1 \mathrm{p}$ shell) and $(3,3)(1 \mathrm{~d} 2 \mathrm{~s}$ shell). Even though proton-unstable nuclei are not included in the comparison, deviations from zero of about $-40 \mathrm{keV}$ are apparent. These deviations are believed to represent the Thomas-Ehrman shift ${ }^{29}$ ) which is inherent to unbound as well as weakly bound states. Reduced proton binding energies lead to increased charge radii, hence slightly lower Coulomb energies.

The standard deviation of $45 \mathrm{keV}$ (table 2) achieved in the global procedure (B) can be compared to previous work. Equally good agreement using similar equations has been obtained before ${ }^{18}$ ) for light nuclei where protons and neutrons occupy the same major shell regions. A Coulomb energy equation ${ }^{30}$ ) for spherical nuclei with diffuse surfaces including exchange and the electromagnetic spin-orbit interaction yielded a standard deviation of $21 \mathrm{keV}$ for 42 spherical nuclei with $Z \geqslant 28$. Charge-dependent nuclear forces and other small effects were included phenomenologically. Deformed nuclei were observed to deviate systematically. Simple two-parameter equations are known ${ }^{18}$ ) to provide a surprisingly good global description of Coulomb displacement energies. Indeed, the equations

$$
\begin{aligned}
& \Delta E_{\mathrm{C}}=a_{1} Z_{<} / A^{1 / 3}-b_{1}, \\
& \Delta E_{\mathrm{C}}=a_{2}\left(Z_{<}+0.5\right) / A^{1 / 3}-b_{2}, \\
& \Delta E_{\mathrm{C}}=\left(a_{3} Z_{<}-b_{3}\right) / A^{1 / 3},
\end{aligned}
$$

with

$$
\begin{array}{ll}
a_{1}=1408 \mathrm{keV}, & b_{1}=641 \mathrm{keV}, \\
a_{2}=1422 \mathrm{keV}, & b_{2}=939 \mathrm{keV}, \\
a_{3}=1375 \mathrm{keV}, & b_{3}=1492 \mathrm{keV},
\end{array}
$$

yield $\sigma_{1}=73 \mathrm{keV}, \sigma_{2}=77 \mathrm{keV}$ and $\sigma_{3}=81 \mathrm{keV}$ which is to be compared to $\sigma=43 \mathrm{keV}$ of table 2 (288 cases). The mean values for the differences between experimental and calculated ground-state masses of higher-order mirror nuclei $\left(T_{z}= \pm T\right.$ with $\left.T>\frac{1}{2}\right)$ are $\left\langle\varepsilon_{1}\right\rangle=-28 \pm 150 \mathrm{keV},\left\langle\varepsilon_{2}\right\rangle=-56 \pm 151 \mathrm{keV}$ and $\left\langle\varepsilon_{3}\right\rangle=$ $+8 \pm 192 \mathrm{keV}$ which is to be compared to $\langle\varepsilon\rangle=-38 \pm 93 \mathrm{keV}$ of table 3 (36 cases). A similar evaluation of Coulomb energy equations used as part of atomic mass equations has been performed recently by Zeldes et al. ${ }^{25}$ ). Standard deviations in the range $120 \mathrm{keV}$ to $1600 \mathrm{keV}$ were found. It is concluded that the Coulomb energy equations with parameters from this work are significantly better than other equations used in global analyses of Coulomb displacement energies. They are also expected to provide realistic estimates of total ground-state Coulomb energies.

The interaction energies $\Omega(Z, N)=S(Z, N) \omega$ from the global procedure (B) which are plotted in fig. 6 for nuclei along the line of $\beta$-stability display discrete discontinuities at proton shell crossings. Neutron shell crossings have only a minor 
effect due to the change in size factor. As mentioned earlier, the core-core interaction energies $\Omega_{\mathrm{cc}}$ at the beginning of each proton shell region are equal to an appropriate sum of all four interaction energies at the end of the preceding shell region. For $A>40$ the data yield $\Omega_{\mathrm{cc}}>\Omega_{\mathrm{cv}}>\Omega_{\mathrm{vv}}$ which is normally expected since valence protons occupy orbits with higher harmonic oscillator quantum numbers, hence increased radii. The ordering is different in the two lower major shell regions: $\Omega_{\mathrm{cc}}>\Omega_{\mathrm{vv}}>\Omega_{\mathrm{cv}}$ for the $1 \mathrm{p}$ shell and $\Omega_{\mathrm{vv}}>\Omega_{\mathrm{cc}}>\Omega_{\mathrm{cv}}$ for the $1 \mathrm{~d} 2 \mathrm{~s}$ shell. An explanation of these results can possibly be found from a detailed quantum mechanical analysis using realistic interactions. This was beyond the scope of the present work. However, estimates including exchange effects have been made for the two lowest shells using harmonic oscillator wave functions. Indeed, $\left.\left.\left\langle(1 s)^{2}\left|r^{-1}\right|(1 s)^{2}\right\rangle\right\rangle\left\langle(1 p)^{2}\left|r^{-1}\right|(1 p)^{2}\right\rangle\right\rangle$ $\left\langle 1 \mathrm{~s}, 1 \mathrm{p}\left|r^{-1}\right| 1 \mathrm{~s}, 1 \mathrm{p}\right\rangle$ is found which confirms the ordering for the $1 \mathrm{p}$ shell. The large value for $\Omega_{\mathrm{v}}$ in the $1 \mathrm{~d} 2 \mathrm{~s}$ shell which involves interactions among the $1 \mathrm{~d}_{5 / 2}, 2 \mathrm{~s}_{1 / 2}$ and $1 d_{3 / 2}$ valence protons could not be confirmed. This may be related to relatively largc exchange integrals within the lower shells and between the valence and the lower shells.

The interaction energy $\Omega_{\mathrm{v}}$ in the shell region $(7,7)$ is exceptionally small. Whereas this could have an underlying physical reason such as the onset of fission and increased instability towards $\alpha$-decay, it is more likely just fortuitous and due to the limited data which made it necessary to include all data with $Z, N \geqslant 82$ in a single shell region. The global analysis $(B)$ was repeated with the constraint that in region $(7,7)$ the energy $\Omega_{\mathrm{v} v}$ should not be smaller than $0.93 \Omega_{\mathrm{cv}}$. Only $\Omega_{\mathrm{vv}}$ changes significantly. These values are included in table 1 and fig. 6 . They are more "reasonable," but the differences $\varepsilon$ for ${ }^{232} \mathrm{Th}$ and ${ }^{238} \mathrm{U}$ are about $-270 \mathrm{keV}$. This is more negative than expected for the known deformations. More data in this region are needed to better establish the significance of these observations.

All exponents $\sigma_{i}$ in the size factors are less than unity, and the interaction energies $\Omega$ therefore decrease less rapidly than $A^{-1 / 3}$ within the shell regions. However, the overall decrease is not too different from the $A^{-1 / 3}$ dependence as can be seen from the comparison with the dotted line in fig. 6 . This behavior may explain why equations based on liquid drop models are successful in describing the general characteristics of Coulomb energies.

The results from this work have several important applications. Excitation energies of IAS can be predicted and compared to new data. The coefficients of the isobaric multiplet mass equation can be predicted and compared to new data. The Coulomb matrix elements of this work can be compared to theoretical calculations. They can also be used to separate combined nuclear and Coulomb interaction energies into the two components.

Another important application is related to the prediction of masses and binding energies of nuclear ground states. For any known neutron-rich nucleus, the mass of the proton-rich (higher-order) mirror nucleus is directly obtained by adding the appropriate Coulomb energy difference. These predictions are subject to the 
Thomas-Ehrman shift ${ }^{29}$ ), though, which has to be estimated independently. Coulomb energy expressions are an integral part of mass equations. Procedures where the present Coulomb energy expressions might be particularly useful are the shell-model equations of Zeldes ${ }^{25,26}$ ), liquid-drop-model equations ${ }^{31}$ ), and the method based on inhomogeneous partial difference equations ${ }^{32,19}$ ). Subtracting the Coulomb energy contributions from the experimental binding energies makes it possible and mandatory to use charge-symmetric expressions to describe the nuclear contributions.

\section{Summary}

Shell-model Coulomb energy equations have been used to describe the experimental Coulomb displacement energies of about 288 nuclei with $4<A<240$. A standard deviation of $\sigma=45 \mathrm{keV}$ has been obtained. Shell effects are apparent including the influence of neutron configurations on the Coulomb energy in the isobaric analog state. The influence of the deformed nuclear shape in the regions $A \approx 100$ and $A \approx 166$ is recognizable. The dependence of the Coulomb interaction energies between valence-valence, valence-core and core-core protons on the number of nucleons is discussed for the various shell regions. They decrease less rapidly than $A^{-1 / 3}$, and the core-core interaction energy is generally the strongest. Important applications for the shell-model Coulomb energy equations include their use in mass and binding energy equations.

Discussions with K.T. Hecht are highly appreciated. This work was supported in part by the US National Science Foundation and the United States-Israel Binational Science Foundation.

\section{References}

1) C.F. von Weizsäcker, Z. Phys. 96 (1935) 431

2) H.A. Bethe and R.F. Bacher, Rev. Mod. Phys. 8 (1936) 82

3) H.A. Bethe, Phys. Rev. 54 (1938) 436

4) J.D. Anderson and C. Wong, Phys. Rev. Lett. 7 (1961) 250

5) J.D. Fox, C.F. Moore and D. Robson, Phys. Rev. Lett. 12 (1964) 198

6) J. Jänecke, E.H.L. Aarts, A.G. Drentje, M.N. Harakeh and C. Gaarde, Nucl. Phys. A394 (1983) 39

7) J. Jänecke, F.D. Becchetti, W.S. Gray, R.S. Tickle and E. Sugarbaker, Nucl. Phys. A402 (1983) 262; J. Jänecke and F.D. Becchetti, Phys. Rev. C27 (1983) 2282

8) B.C. Carlson and I. Talmi, Phys. Rev. 96 (1954) 436

9) K.T. Hecht, Nucl. Phys. A102 (1967) 11

10) K.T. Hecht, Nucl. Phys. A114 (1968) 280

11) J.A. Nolen and J.P. Schiffer, Ann. Rev. Nucl. Sci. 19 (1969) 471

12) N. Auerbach, J. Hüfner, A.K. Kerman and C.M. Shakin, Rev. Mod. Phys. 44 (1972) 48

13) E.P. Wigner, in Proc. Robert A. Welch Found. Conf. on chemical research, Houston, Texas, 1957. ed. W.O. Milligan, vol. 1 (The Robert A. Welch Foundation, Houston, Texas, 1958) p. 88

14) S. Weinberg and S.B. Treiman, Phys. Rev. 116 (1958) 465 
15) D.H. Wilkinson, Phys. Lett. 11 (1964) 243; 12 (1964) 348; Phys. Rev. Lett. 13 (1964) 571

16) W. Benenson and E. Kashy, Rev. Mod. Phys. 51 (1980) 527

17) A. de-Shalit and I. Talmi, Nuclear shell theory (Academic Press, New York, 1963)

18) J. Jänecke, in Isospin in nuclear physics, ed. D.H. Wilkinson (North-Holland, Amsterdam, 1969) ch. 8

19) J. Jänecke, in Atomic masses and fundamental constants 6 (Plenum, New York, 1980) p. 101

20) R. Sherr and G. Bertsch, Phys. Rev. C12 (1975) 1671

21) W.J. Courtney and J.D. Fox, At. Data and Nucl. Data Tables 15 (1975) 141

22) A.H. Wapstra, 1981 Midstream Mass Evaluation

23) F.D. Becchetti, W.S. Gray, J. Jänecke, E.R. Sugarbaker and R.S. Tickle, Nucl. Phys. A271 (1976) 77

24) Fortran Program ZXSSQ, International Mathematical Statistical Library (IMSL)

25) N. Zeldes, A. Novoselsky and A. Taraboulos, Niels Bohr Institute Report NBI-82-11, to be published

26) S. Liran and N. Zeides, At. Data and Nucl. Data Tables 17 (1975) 431

27) E. Comay and I. Kelson, At. Data and Nucl. Data Tables 17 (1975) 463

28) E. Comay and I. Kelson, Z. Phys. 310 (1983) 107

29) R.G. Thomas, Phys. Rev. 81 (1951) 148; 88 (1952) 1109;

J.B. Ehrman, Phys. Rev. 81 (1951) 412;

A.M. Lane and R.G. Thomas, Rev. Mod. Phys. 30 (1958) 257

30) J. Jänecke, Nucl. Phys. A181 (1972) 49

31) W.D. Meyers, Droplet model of atomic nuclei (EFI/Plenum, New York, 1977)

32) J. Jänecke, At. Data and Nucl. Data Tables 17 (1975) 455 